\title{
Excited Muon Searches at the FCC-Based Muon-Hadron Colliders
}

\author{
A. Caliskan, ${ }^{1}$ S. O. Kara, $^{2}$ and A. Ozansoy ${ }^{3}$ \\ ${ }^{1}$ Faculty of Engineering and Natural Sciences, Department of Physics Engineering, Gümüşhane University, 29100 Gümüşhane, Turkey \\ ${ }^{2}$ Omer Halisdemir University, Bor Vocational School, 51240 Nigde, Turkey \\ ${ }^{3}$ Faculty of Sciences, Department of Physics, Ankara University, Tandogan, 06100 Ankara, Turkey
}

Correspondence should be addressed to A. Caliskan; acaliskan@gumushane.edu.tr

Received 8 February 2017; Revised 8 March 2017; Accepted 13 March 2017; Published 30 March 2017

Academic Editor: Luca Stanco

Copyright (C) 2017 A. Caliskan et al. This is an open access article distributed under the Creative Commons Attribution License, which permits unrestricted use, distribution, and reproduction in any medium, provided the original work is properly cited. The publication of this article was funded by $\mathrm{SCOAP}^{3}$.

\begin{abstract}
We study the excited muon production at the FCC-based muon-hadron colliders. We give the excited muon decay widths and production cross-sections. We deal with the $\mu p \rightarrow \mu^{\star} q \rightarrow \mu \gamma q$ process and plot the transverse momentum and normalized pseudorapidity distributions of final state particles to define the kinematical cuts best suited for discovery. By using these cuts, we get the mass limits for excited muons. It is shown that the discovery limits obtained on the mass of $\mu^{\star}$ are $2.2,5.8$, and $7.5 \mathrm{TeV}$ for muon energies of 63,750 , and $1500 \mathrm{GeV}$, respectively.
\end{abstract}

\section{Introduction}

Discovery of the Higgs boson by ATLAS and CMS collaborations in $2012[1,2]$ has proved the accuracy and reliability of the Standard Model (SM) of the particle physics. But, many questions about dark matter, supersymmetric particles, extra dimensions, neutrino masses, asymmetry between matter and antimatter, existence of new fundamental interactions, and fermion substructure are keeping their mystery and waiting to be solved. Many theories beyond the SM (BSM) have been proposed for these puzzling phenomena. Evidently, it is necessary to perform the particle physics experiments in more powerful colliders with higher energies and luminosities.

Compositeness is one of the BSM models that intend to solve the problem of fermionic families replication, by introducing more fundamental matter constituents called preons. Excited fermions are predicted by preonic models and their existence would be a strong evidence for fermion substructure [3-5]. If known quarks and leptons present composite structures, reasonable explanations could be given for the still unanswered questions about the number and replication of SM families and their mass hierarchy. The appearance of excited states is an indisputable consequence of composite structure of known fermions [6-9]. In composite models, SM fermions are considered as ground states of a rich and heavier spectrum of excited states. Charged $\left(e^{\star}, \mu^{\star}, \tau^{\star}\right)$ and neutral $\left(v_{e}^{\star}, v_{\mu}^{\star}, v_{\tau}^{\star}\right)$ excited leptons come on the scene in the framework of composite models. Excited leptons with spin-1/2 and weak-isospin-1/2 are considered as the lowest radial and orbital excitations. Excited states with higher spins also appear in composite models [10-14].

Considerable searches for the spin-1/2 charged and neutral excited lepton signatures have been performed for the $e^{+} e^{-}$and $e p$ colliders [15-18]; $\gamma \gamma$ [19-22] and $e \gamma[14,23]$ colliders; $p p$ [24-27] and $p \bar{p}[28-30]$ colliders. Production and decay properties of spin-1/2 excited leptons in a left-right symmetric scenario are studied in [31]. Also, spin-3/2 excited leptons are studied at various colliders in [32-38].

Excited electrons $\left(e^{\star}\right)$ are extensively investigated in the field of excited leptonic state studies. To perform a main 
TABLE 1: Main parameters of the FCC-based $\mu p$ collider.

\begin{tabular}{lccr}
\hline Collider & $E_{\mu}(\mathrm{TeV})$ & $\sqrt{s}(\mathrm{TeV})$ & $L_{\mu p}\left(\mathrm{~cm}^{-2} \mathrm{~s}^{-1}\right)$ \\
\hline$\mu 63$-FCC & 0.063 & 3.50 & $0.2 \times 10^{31}$ \\
$\mu 750$-FCC & 0.75 & 12.2 & $50 \times 10^{31}$ \\
$\mu 1500$-FCC & 1.5 & 17.3 & $50 \times 10^{31}$ \\
\hline
\end{tabular}

comparison it is necessary to study the other charged excited leptons $\left(\mu^{\star}\right.$ and $\left.\tau^{\star}\right)$. In principle, $\mu^{\star}$ and $\tau^{\star}$ contributions would differ from $e^{\star}$ contribution in the mass and decay products of the SM leptons.

The mass limit for excited spin-1/2 muons obtained from their pair production $\left(e^{+} e^{-} \rightarrow \mu^{+\star} \mu^{-\star}\right)$ by OPAL collaboration at $\sqrt{s}=189-209 \mathrm{GeV}$ is $m_{\mu^{*}}>103.2 \mathrm{GeV}$ [39]. From single production $\left(p p \rightarrow \mu \mu^{\star} X\right)$, in events with three or more charged leptons at $\sqrt{s}=8 \mathrm{TeV}$ including contact interactions in the $\mu^{\star}$ production and decay mechanism, the ATLAS collaboration sets the mass limits as $m_{\mu^{*}}>3000 \mathrm{GeV}$ [40]. Other studies on excited muon searches can be found in [4151].

Enormous efforts are being made for the research and development of new particle colliders for the Large Hadron Collider (LHC) era and post-LHC era. A staged approach will be taken into consideration for the planning of these energy frontiers. The first stage is low-energy lepton colliders to make the precision measurements of the LHC discoveries. These projects are the International Linear Collider (ILC) [52] with a center-of-mass energy of $0.5 \mathrm{TeV}$ and low-energy muon collider $\left(\mathrm{a} \mu^{+} \mu^{-}\right.$collider, shortly $\left.\mu \mathrm{C}\right)$ [53]. Lepton-hadron collider projects would be considered as a second stage, including an ep collider under design, namely, Large Hadron Electron Collider ( $\mathrm{LHeC}$ ) with $\sqrt{s}=1.3 \mathrm{TeV}$ (possibly upgraded to $\sqrt{s}=1.96 \mathrm{TeV})[54,55]$, and a hypothetical $\mu p$ collider $\mu$-LHC at this stage. The ILC with an increased center-of-mass energy $(\sqrt{s}=1 \mathrm{TeV})$, the Compact Linear Collider (CLIC) [56] with an optimal center-of-mass energy of $3 \mathrm{TeV}$, and the Plasma Wake-Field Accelerator-Linear Collider project (PWFA-LC) [57] are high-energy linear $e^{+} e^{-}$ colliders under consideration to be built after the LHC. On the side of muon colliders, $\mu \mathrm{C}$ with $\sqrt{s}$ up to $3 \mathrm{TeV}$ is planned as a high-energy muon collider [53].

The Future Circular Collider (FCC) [58] project investigates the various concepts of the circular colliders at CERN for the post-LHC era. The FCC is proposed as the future $p p$ collider with $\sqrt{s}=100 \mathrm{TeV}$ and supported by the European Union within the Horizon 2020 Framework Programme for research and innovation. Besides the $p p$ option, it is also being planned to include the $e^{+} e^{-}$collider option (TLEP or FCC-ee) [59] and several ep collider options [60, 61].

Building a muon collider as a dedicated $\mu$-ring tangential to the FCC will give opportunity to handle multi-TeV scale $\mu p$ and $\mu A$ colliders $[62,63]$. Assumed values for muon energy, center-of-mass energy, and average instantaneous luminosity for different FCC-based $\mu p$ collider options are given in Table 1.

Excited muon searches would provide complementary information for the compositeness studies. This work is dedicated to the search for excited muons at future FCCbased muon-proton colliders. We introduce the effective Lagrangian responsible for the gauge interactions of excited muons and give their decay widths in Section 2. Production cross-sections and the analysis for the $\mu^{\star} \rightarrow \mu \gamma$ decay mode are presented in Section 3. We summarized our results in Section 4.

\section{Effective Lagrangian}

A spin-1/2 excited lepton is the lowest radial and orbital excitation according to the classification by $S U(2) \times U(1)$ quantum numbers. Interactions between excited spin-1/2 leptons and ordinary leptons are of magnetic transition type $[15,16,64]$. The effective Lagrangian for the interaction between a spin-1/2 excited lepton, a gauge boson $(V=$ $\left.\gamma, Z, W^{ \pm}\right)$, and the SM lepton is given by

$$
L=\frac{1}{2 \Lambda} \overline{l_{R}^{*}} \sigma^{\mu \nu}\left[f g \frac{\vec{\tau}}{2} \cdot \vec{W}_{\mu \nu}+f^{\prime} g^{\prime} \frac{Y}{2} B_{\mu \nu}\right] l_{L}+\text { h.c. }
$$

where $\Lambda$ is the new physics scale, $W_{\mu \nu}$ and $B_{\mu \nu}$ are the field strength tensors, $\vec{\tau}$ denotes the Pauli matrices, $Y$ is the hypercharge, $g$ and $g^{\prime}$ are the gauge couplings, and $f$ and $f^{\prime}$ are the scaling factors for the gauge couplings of $S U(2)$ and $U(1) ; \sigma^{\mu \nu}=i\left(\gamma^{\mu} \gamma^{\nu}-\gamma^{\nu} \gamma^{\mu}\right) / 2$ with $\gamma^{\mu}$ being the Dirac matrices. An excited lepton has three possible decay modes: radiative decay $l^{\star} \rightarrow l \gamma$, neutral weak decay $l^{\star} \rightarrow l Z$, and charged weak decay $l^{\star} \rightarrow \nu W$. Neglecting the SM lepton mass, we find the decay width of excited leptons as

$$
\Gamma\left(l^{\star} \longrightarrow l V\right)=\frac{\alpha m^{\star 3}}{4 \Lambda^{2}} f_{V}^{2}\left(1-\frac{m_{V}^{2}}{m^{\star 2}}\right)^{2}\left(1+\frac{m_{V}^{2}}{2 m^{\star 2}}\right),
$$

where $f_{V}$ is the new electroweak coupling parameter corresponding to the gauge boson $V$, and $f_{\gamma}=-\left(f+f^{\prime}\right) / 2$, $f_{Z}=\left(-f \cot \theta_{W}+f^{\prime} \tan \theta_{W}\right) / 2$, and $f_{W}=f / \sqrt{2} \sin \theta_{W} ; \theta_{W}$ is the weak mixing angle, $m_{V}$ is the mass of the gauge boson, and $m^{\star}$ is the mass of the excited lepton. Total decay widths of excited leptons for $\Lambda=m^{\star}$ and $\Lambda=100 \mathrm{TeV}$ are given in Figure 1.

\section{Excited Muon Production at $\mu p$ Colliders}

The FCC-based $\mu p$ colliders will provide the potential reach for excited muon searches through the $\mu p \rightarrow \mu^{\star} X$ process. Feynman diagrams for the subprocesses $\mu q(\bar{q}) \rightarrow \mu^{\star} q(\bar{q})$ are shown in Figure 2. We implemented excited muon interaction vertices in high-energy physics simulation programme CALCHEP [65-67] and used it in our calculations. 


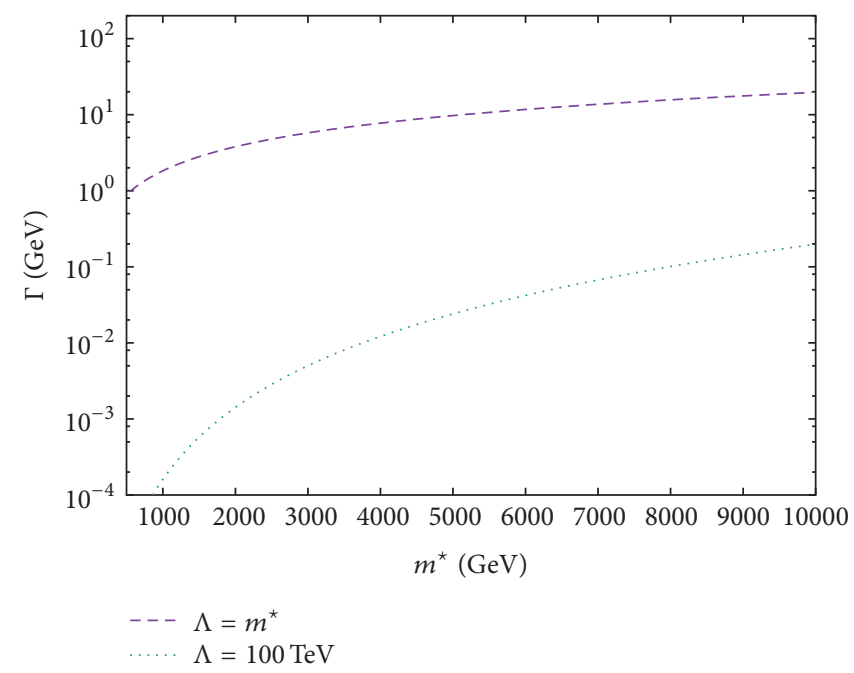

FIGURE 1: Decay width of excited leptons for $\Lambda=m^{\star}$ and $\Lambda=100 \mathrm{TeV}$.
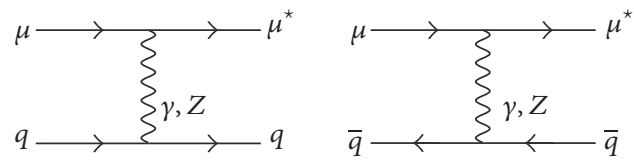

FIgURE 2: Leading-order Feynman diagrams for the $\mu^{\star}$ production at $\mu p$ collider.

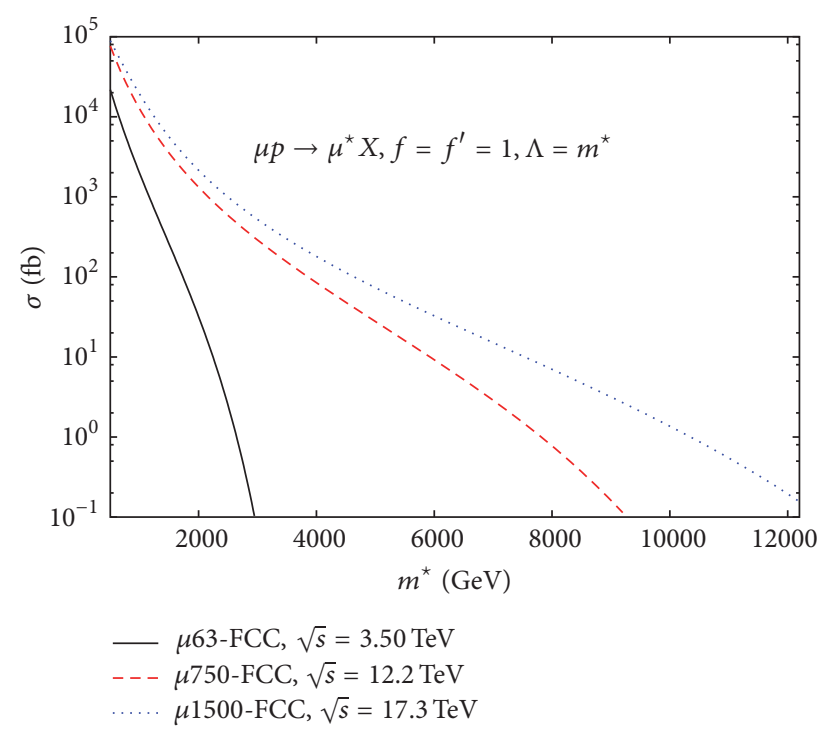

(a)

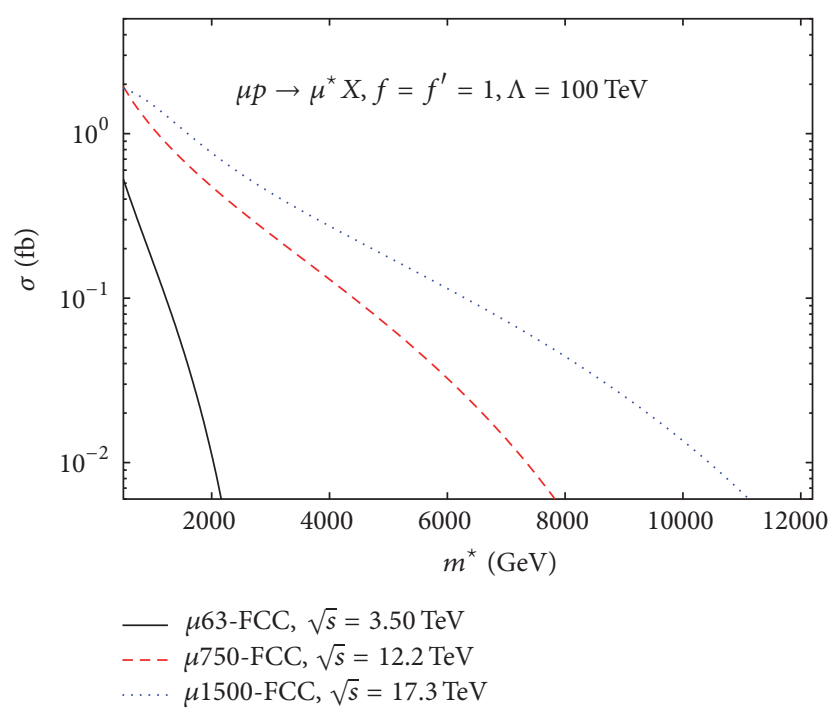

(b)

FIGURE 3: Total cross-section as a function of the excited muon mass for the $\mu p$ colliders with various center-of-mass energies for $\Lambda=m^{*}$ (a) and $\Lambda=100 \mathrm{TeV}(\mathrm{b})$, respectively.

The total cross-section for the process $\mu p \rightarrow \mu^{\star} X$ as a function of the excited muon mass is shown in Figure 3. We used the CTEQ6L parton distribution function in our calculations.

For the analysis we take into account the $\mu \gamma$ decay mode of the $\mu^{\star}$. We deal with the process $\mu p \rightarrow \mu^{\star} X \rightarrow \mu \gamma X$ (subprocess $\mu q(\bar{q}) \rightarrow \mu \gamma q(\bar{q})$ ) and impose generic cuts, $p_{T}>$ $20 \mathrm{GeV}$, for the final state muon, photon, and jets.

Standard Model cross-sections after the application of the generic cuts are $\sigma_{B}=24.51 \mathrm{pb}, \sigma_{B}=89.69 \mathrm{pb}$, and $\sigma_{B}=$ $122.43 \mathrm{pb}$ for $\sqrt{s}=3.50,12.2$, and $17.3 \mathrm{TeV}$, respectively. We show the transverse momentum distributions in Figure 4 (for 
TABLE 2: Discovery cuts.

\begin{tabular}{lcccc}
\hline Collider & $p_{T}^{\mu}$ cut & $p_{T}^{\gamma}$ cut & $\eta^{\mu}$ cut & \multicolumn{1}{c}{$\eta^{\gamma}$ cut } \\
\hline$\mu 63$-FCC & $p_{T}^{\mu}>450 \mathrm{GeV}$ & $p_{T}^{\gamma}>300 \mathrm{GeV}$ & $-4.5<\eta^{\mu}<-0.8$ & $-4.8<\eta^{\gamma}<-1.2$ \\
$\mu 750-$ FCC & $p_{T}^{\mu}>1200 \mathrm{GeV}$ & $p_{T}^{\gamma}>900 \mathrm{GeV}$ & $-3.5<\eta^{\mu}<0.5$ & $-4<\eta^{\gamma}<0.3$ \\
$\mu 1500-$ FCC & $p_{T}^{\mu}>1500 \mathrm{GeV}$ & $p_{T}^{\gamma}>1500 \mathrm{GeV}$ & $-3<\eta^{\mu}<1$ & $-4<\eta^{\gamma}<0.5$ \\
\hline
\end{tabular}

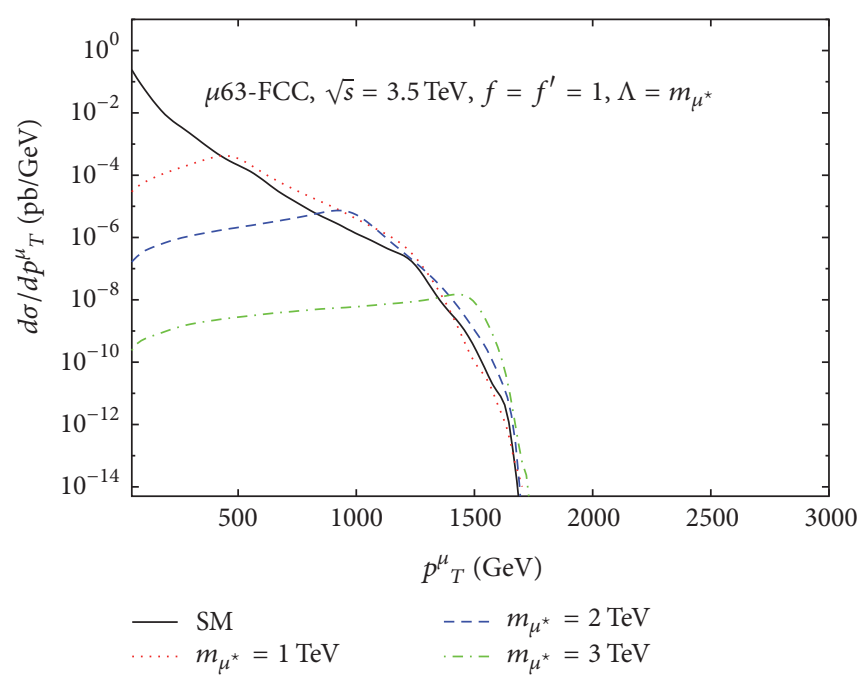

(a)

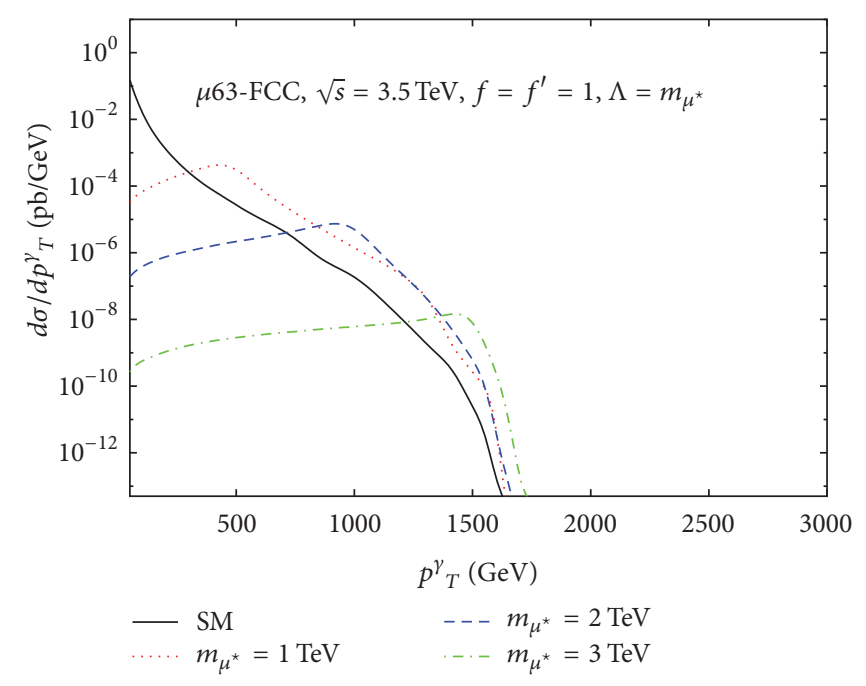

(b)

Figure 4: Muon (a) and photon (b) $p_{T}$ distributions for the $\mu 63$-FCC.

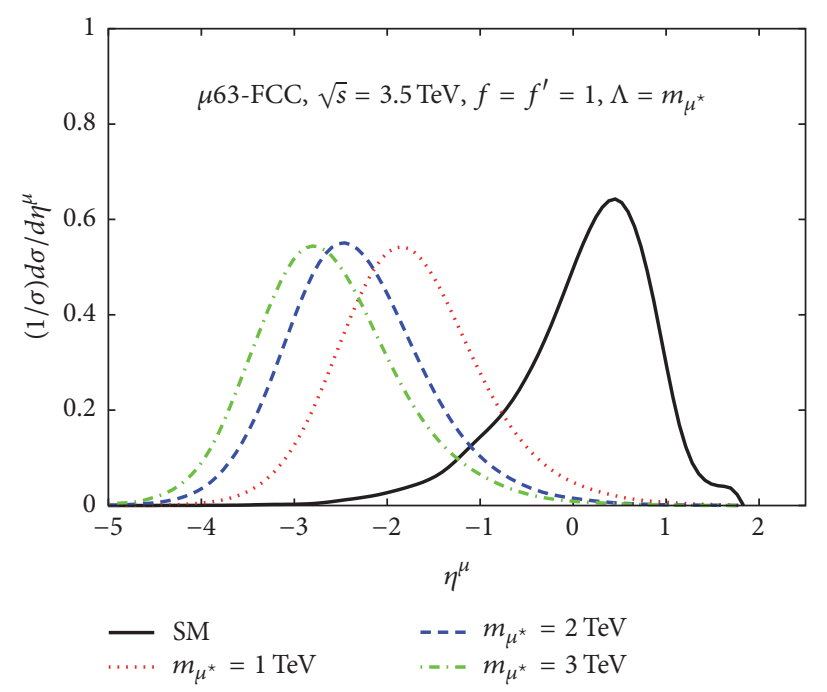

(a)

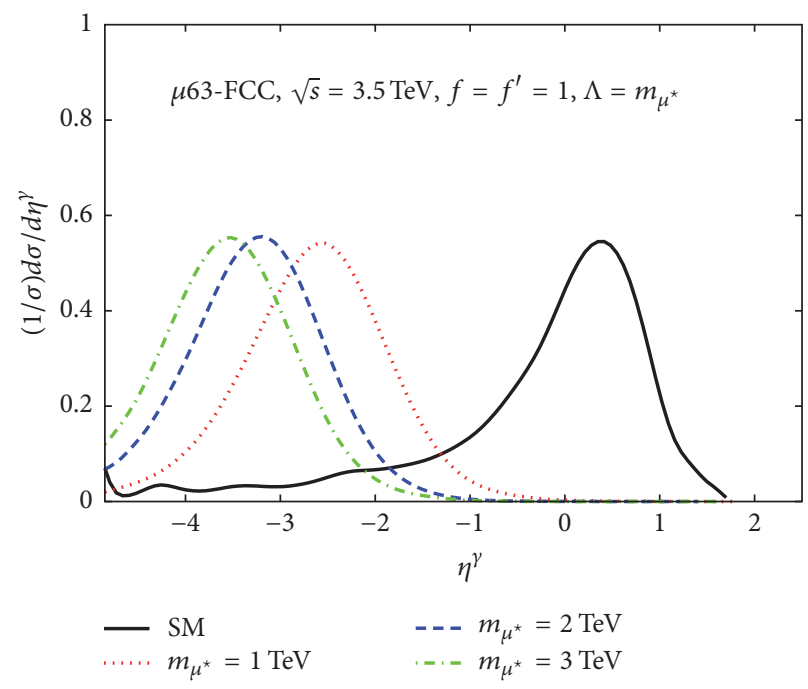

(b)

FIGURE 5: Muon (a) and photon (b) normalized $\eta$ distributions for the $\mu 63$-FCC.

$\mu 63$-FCC), in Figure 6 (for $\mu 750$-FCC), and in Figure 8 (for $\mu 1500-\mathrm{FCC})$; the normalized pseudorapidity distributions are in Figure 5 (for $\mu 63$-FCC), in Figure 7 (for $\mu 750$-FCC), and in Figure 9 (for $\mu 1500-$ FCC). We choose $f=f^{\prime}=1$ and $\Lambda=m_{\mu}^{\star}$ in our calculations. As it is seen from Figures 4,6 , and 8 excited muons carry high transverse momentum and these distributions show a peak around $m_{\mu^{*}} / 2$. Also, normalized pseudorapidity distributions are so asymmetric. Since pseudorapidity is defined to be $\eta=-\ln (\tan (\theta / 2))$, where $\theta$ is the polar angle, it is concluded that excited muons are produced mostly in the backward direction.

By examining these distributions we determine the discovery cuts presented in Table 2. To determine these discovery cuts we specify the optimal regions where we cut 


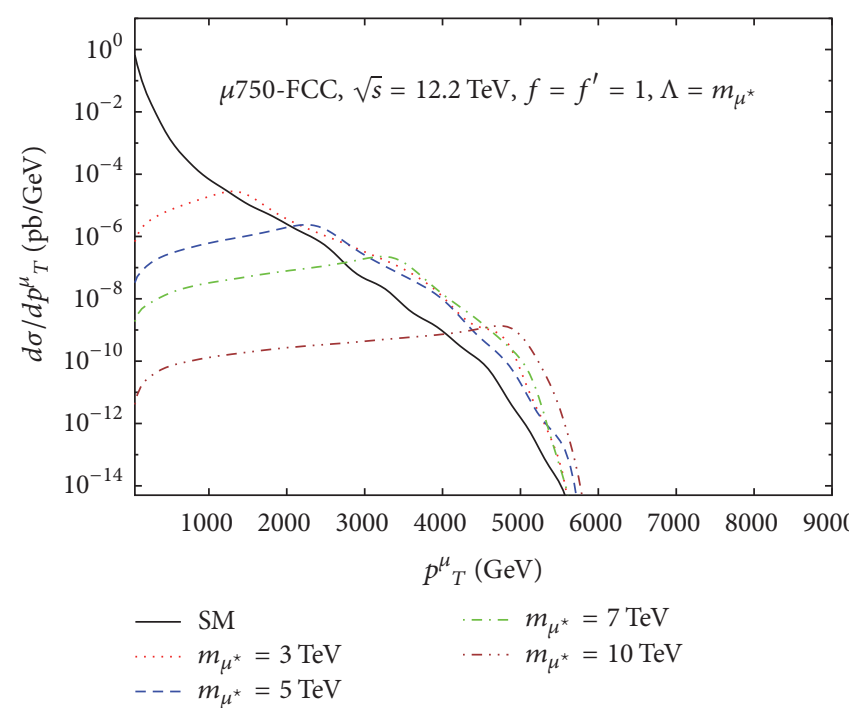

(a)

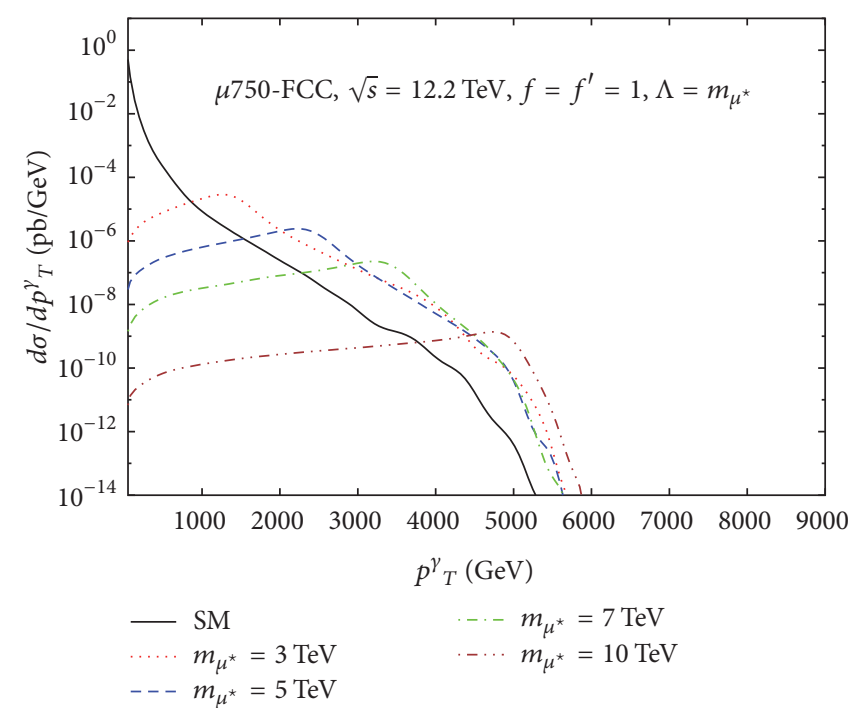

(b)

Figure 6: Muon (a) and photon (b) $p_{T}$ distributions for the $\mu 750$-FCC.

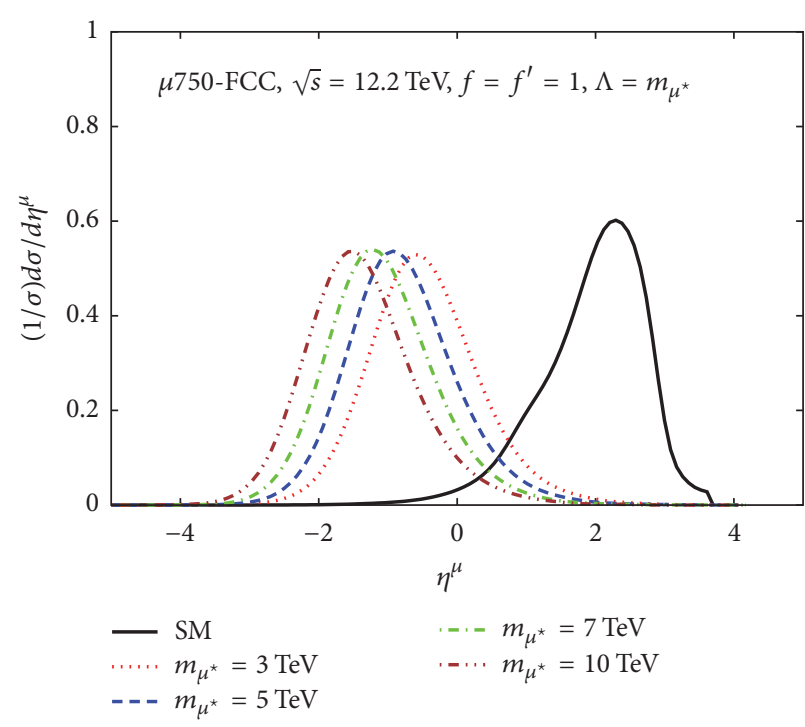

(a)

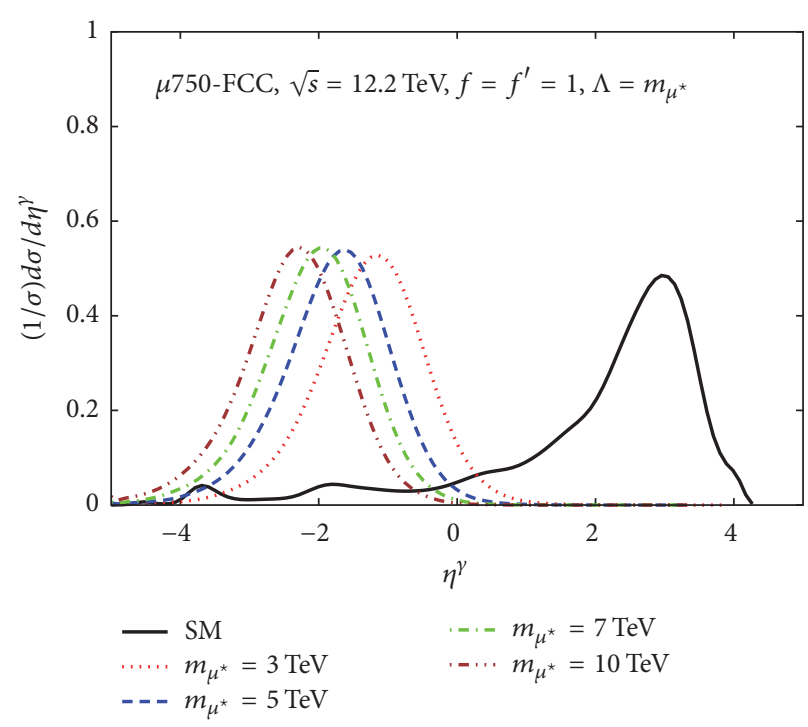

(b)

FIGURE 7: Muon (a) and photon (b) normalized $\eta$ distributions for the $\mu 750$-FCC.

off the most of the background but at the same time do not affect the signal so much. Since we choose the $\mu^{\star} \rightarrow \mu \gamma$ decay mode of the excited muon (try to identify the excited muons through its decay products), no further cut is made on jets.

The invariant mass distributions following these cuts are shown in Figure 10. We define the statistical significance of the expected signal yield as

$$
S S=\frac{\sigma_{S}}{\sqrt{\sigma_{B}}} \sqrt{\epsilon \cdot L_{\text {int }}}
$$

where $\sigma_{S}$ denotes cross-section due to the excited muon production and $\sigma_{B}$ denotes the SM cross-section, $L_{\text {int }}$ is the integrated luminosity of the collider, and $\epsilon$ is the selection efficiency to detect the signal in the chosen channel $(\epsilon$ is assumed to be the same both on signal and on background). Taking into account the criteria $S S>3(95 \% \mathrm{CL})$ and $S S>5$ ( $99 \%$ CL), we derive the mass limits for excited muons. Our results are summarized in Table 3.

\section{Conclusion}

It is shown that the FCC-based muon-proton colliders have a significant potential in excited muon investigations. We have studied the excited muon production and decay in various 


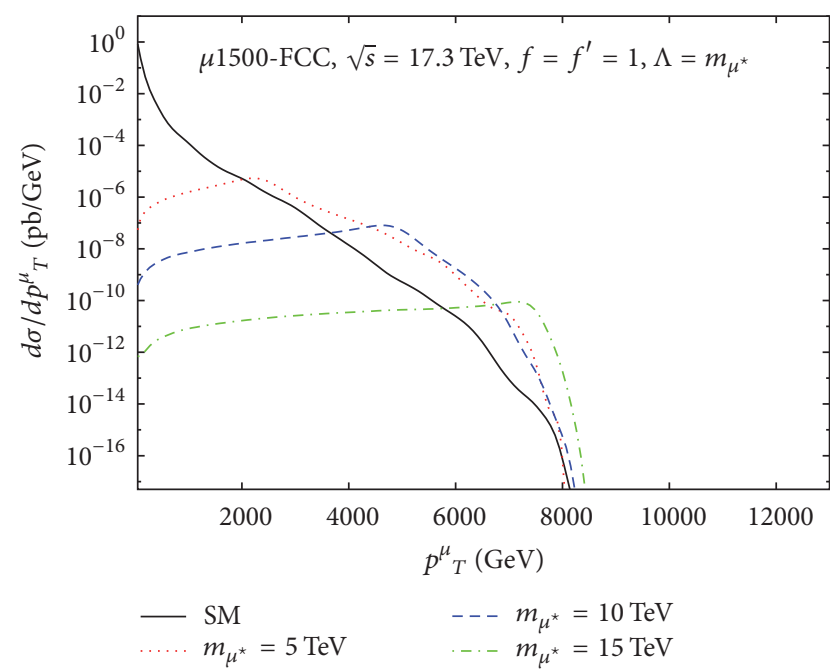

(a)

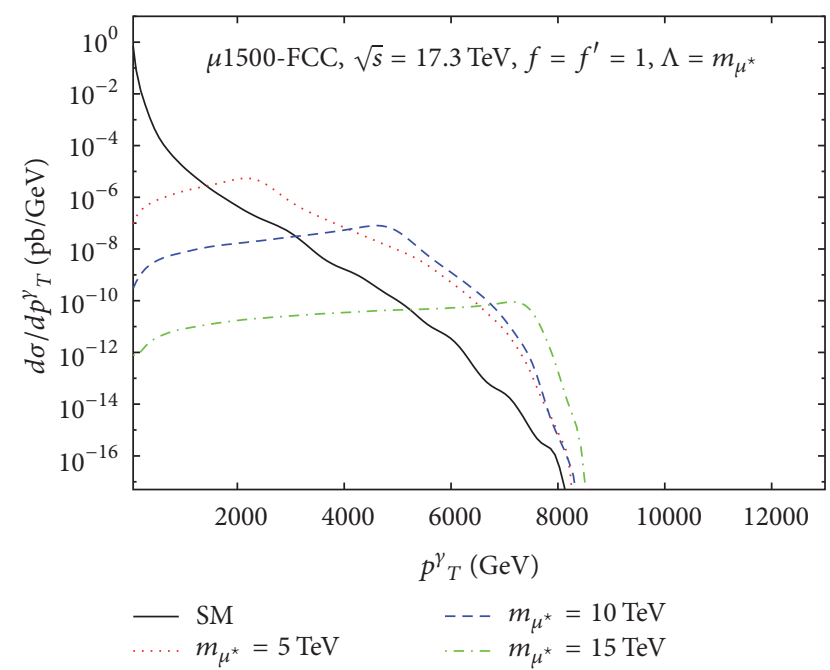

(b)

Figure 8: Muon (a) and photon (b) $p_{T}$ distributions for the $\mu 1500$-FCC.

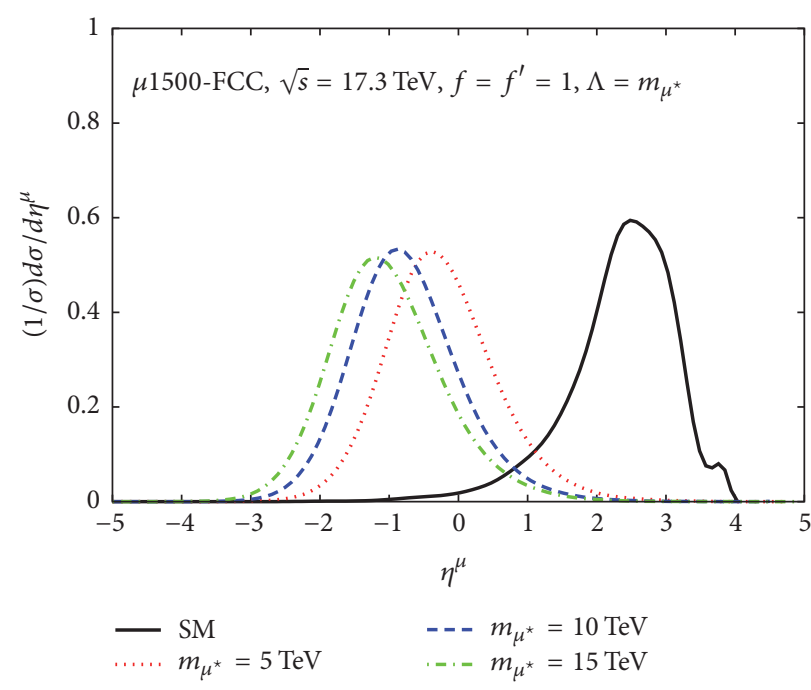

(a)

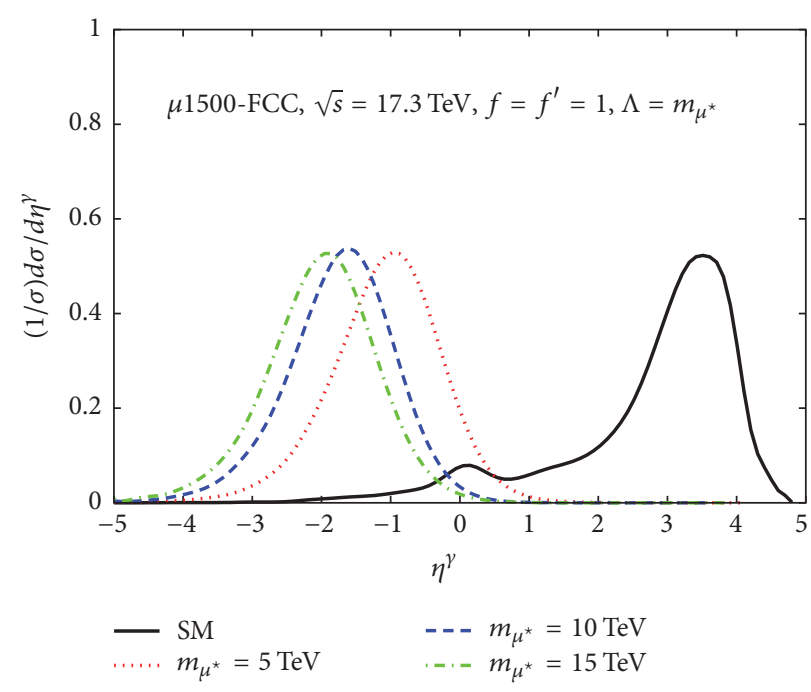

(b)

Figure 9: Muon (a) and photon (b) normalized $\eta$ distributions for the $\mu 750$-FCC $\mu 1500$-FCC.

TABLe 3: Mass limits for $\mu^{\star}$ at FCC-based $\mu p$ colliders.

\begin{tabular}{|c|c|c|c|c|}
\hline \multirow{2}{*}{ Collider } & \multirow{2}{*}{$L_{\mu p}\left(\mathrm{~cm}^{-2} \mathrm{~s}^{-1}\right)$} & \multirow{2}{*}{$\Lambda$} & \multicolumn{2}{|c|}{$m_{\mu^{\star}}(\mathrm{GeV})$} \\
\hline & & & $3 \sigma$ & $5 \sigma$ \\
\hline \multirow{2}{*}{$\mu 63$-FCC } & \multirow{2}{*}{$0.2 \times 10^{31}$} & $m_{\mu^{\star}}$ & 2330 & 2250 \\
\hline & & $100 \mathrm{TeV}$ & 2300 & 2180 \\
\hline \multirow{2}{*}{$\mu 750$-FCC } & \multirow{2}{*}{$50 \times 10^{31}$} & $m_{\mu^{\star}}$ & 6500 & 5950 \\
\hline & & $100 \mathrm{TeV}$ & 6000 & 5830 \\
\hline \multirow{2}{*}{$\mu 1500-\mathrm{FCC}$} & \multirow{2}{*}{$50 \times 10^{31}$} & $m_{\mu^{\star}}$ & 8050 & 7540 \\
\hline & & $100 \mathrm{TeV}$ & 7930 & 7480 \\
\hline
\end{tabular}

FCC-based $\mu p$ collider options with muon energies of 63,750 , and $1500 \mathrm{GeV}$. Our analysis shows that taking into account the $S S>5$ criteria, for $\Lambda=m^{\star}$, excited muon mass limits are $2250 \mathrm{GeV}, 5950 \mathrm{GeV}$, and $7540 \mathrm{GeV}$, for $\sqrt{s}=3.5,12.2$, and $17.3 \mathrm{TeV}$, respectively. Also, for the same criteria, for $\Lambda=100 \mathrm{TeV}$, excited muon mass limits are 2180, 5830, and $7480 \mathrm{GeV}$ for $\sqrt{s}=3.5,12.2$, and $17.3 \mathrm{TeV}$, respectively.

\section{Conflicts of Interest}

The authors declare that they have no conflicts of interest.

\section{Acknowledgments}

A. Caliskan and S. O. Kara's work is supported by the Scientific and Technological Research Council of Turkey (TUBITAK) under the Grant no. 114F337. 

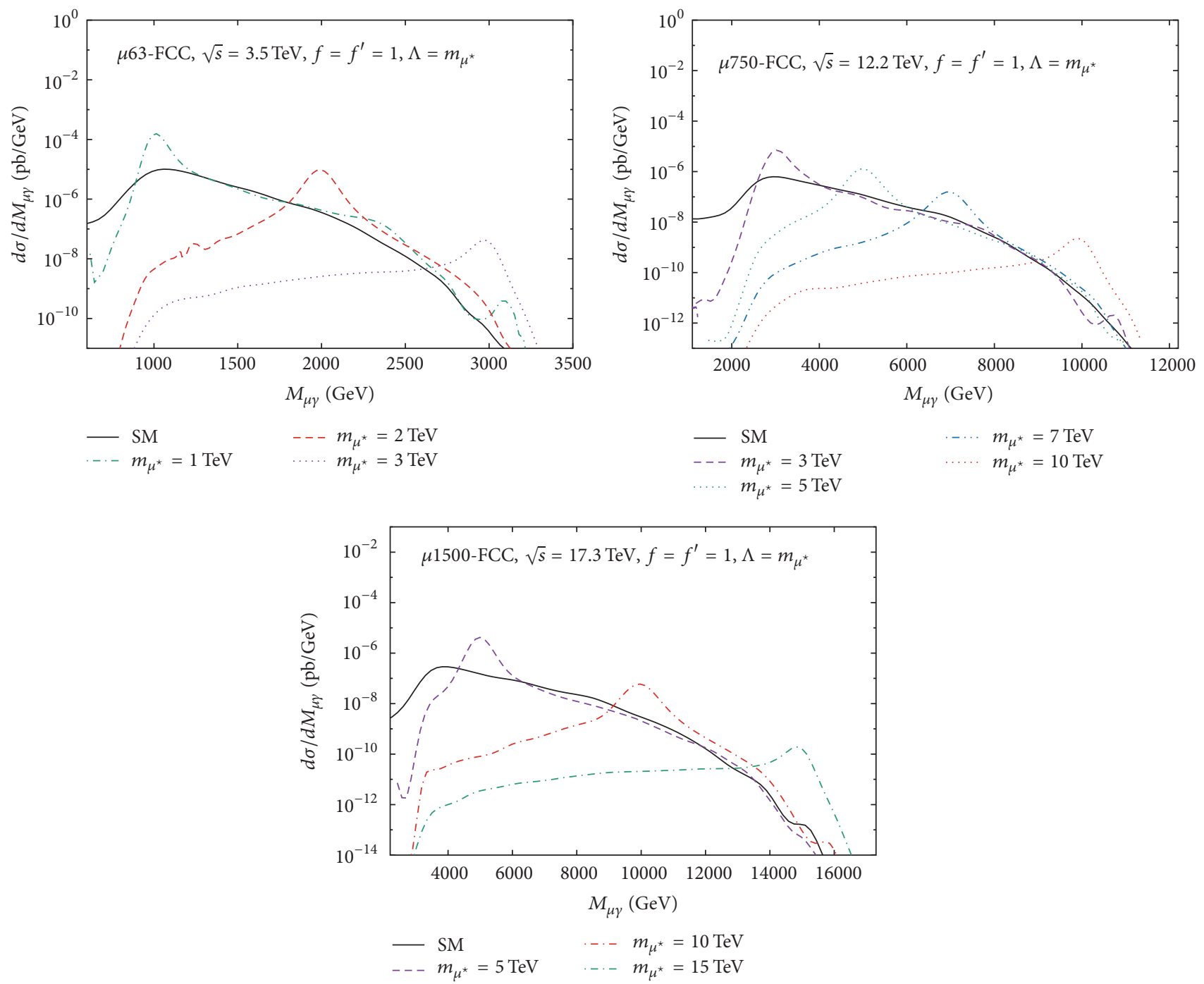

FIGURE 10: Invariant mass distributions of the $\mu \gamma$ system after the discovery cuts for $\mu 63$-FCC, $\mu 750$-FCC, and $\mu 1500$-FCC, respectively.

\section{References}

[1] G. Aad, T. Abajyan, B. Abbott et al., "Observation of a new particle in the search for the Standard Model Higgs boson with the ATLAS detector at the LHC," Physics Letters B, vol. 716, no. 1, pp. 1-29, 2012.

[2] S. Chatrchyan, V. Khachatryan, A. M. Sirunyan et al., "Observation of a new boson at a mass of $125 \mathrm{GeV}$ with the CMS experiment at the LHC," Physics Letters B, vol. 716, no. 1, pp. 30-61, 2012.

[3] H. Terazawa, Y. Chikashige, and K. Akama, "Unified model of the Nambu-Jona-Lasinio type for all elementary-particle forces," Physical Review D, vol. 15, no. 2, pp. 480-487, 1977.

[4] Y. Ne'eman, "Primitive particle model," Physics Letters B, vol. 82, no. 1, pp. 69-70, 1979.

[5] H. Terazawa, M. Yasuè, K. Akama, and M. Hayashi, "Observable effects of the possible sub-structure of leptons and quarks," Physics Letters B, vol. 112, no. 4-5, pp. 387-392, 1982.

[6] F. M. Renard, "Excited quarks and new hadronic states," Il Nuovo Cimento A, vol. 77, no. 1, pp. 1-20, 1983.
[7] E. J. Eichten, K. D. Lane, and M. E. Peskin, "New tests for quark and lepton substructure," Physical Review Letters, vol. 50, no. 11, pp. 811-814, 1983.

[8] A. de Rújula, L. Maiani, and R. Petronzio, "Search for excited quarks," Physics Letters B, vol. 140, no. 3-4, pp. 253-258, 1984.

[9] J. Kühn and P. Zerwas, "Excited quarks and leptons," Physics Letters B, vol. 147, no. 1-3, pp. 189-196, 1984.

[10] J. Leite Lopes, J. A. Martins Simoes, and D. Spehler, "Production and decay properties of possible spin 32 leptons," Physics Letters $B$, vol. 94, no. 3, pp. 367-372, 1980.

[11] J. Leite Lopes, J. A. Martins Simoes, and D. Spehler, "Possible spin-3/2 quarks and scaling violations in neutrino reactions," Physical Review D, vol. 23, no. 3, article 797, 1981.

[12] J. L. Lopes, D. Spehler, and J. A. Simões, "Weak interactions involving spin-3/2 leptons," Physical Review D, vol. 25, no. 7, pp. 1854-1859, 1982.

[13] Y. Tosa and R. E. Marshak, "Exotic fermions," Physical Review $D$, vol. 32, no. 3, pp. 774-780, 1985. 
[14] O. J. P. Éboli, E. M. Gregores, J. C. Montero, S. F. Novaes, and D. Spehler, "Excited leptonic states in polarized $e^{-} \gamma$ and $e^{+} e^{-}$ collisions," Physical Review D, vol. 53, no. 3, pp. 1253-1263, 1996.

[15] K. Hagiwara, S. Komamiya, and D. Zeppenfeld, "Excited lepton production at LEP and HERA," Zeitschrift für Physik C Particles and Fields, vol. 29, no. 1, pp. 115-122, 1985.

[16] F. Boudjema, A. Djouadi, and J. L. Kneur, "Excited fermions at e+e- and eP colliders," Zeitschrift für Physik C Particles and Fields, vol. 57, no. 3, pp. 425-449, 1993.

[17] O. Çakır, A. Yılmaz, and S. Sultansoy, "Single production of excited electrons at future $\mathrm{e}^{+} \mathrm{e}^{-}$, ep and pp colliders," Physical Review D, vol. 70, no. 7, Article ID 075011, 2004.

[18] O. Çakır, İ. Türk Çakır, and Z. Kırca, "Single production of excited neutrinos at future $\mathrm{e}^{+} \mathrm{e}^{-}, e p$ and $p p$ colliders," Physical Review D, vol. 70, no. 7, Article ID 075017, 2004.

[19] I. F. Ginzburg and D. Yu. Ivanov, "Excited leptons and quarks at $\gamma \gamma / \gamma$ e colliders," Physics Letters B, vol. 276, no. 1-2, pp. 214-218, 1992.

[20] A. Belyaev, E. Boos, and A. Pukhov, "Study of excited neutrino production in $e^{+} e^{-}, \gamma e$ and $\gamma \gamma$ collisions at TeV energies," Physics Letters B, vol. 296, no. 3-4, pp. 452-457, 1992.

[21] M. Köksal, "Analysis of excited neutrinos at the CLIC," International Journal of Modern Physics A, vol. 29, no. 24, Article ID 1450138, 2014.

[22] A. Ozansoy and A. A. Billur, "Search for excited electrons through $\gamma$ scattering," Physical Review D, vol. 86, no. 5, Article ID 055008, 2012.

[23] Z. Kirca, O. Çakir, and Z. Z. Aydin, "Production of excited electrons at TESLA and CLIC based e gamma colliders," Acta Physica Polonica B, vol. 34, no. 8, article 4079, 2003.

[24] O. J. Éboli, S. M. Lietti, and P. Mathews, "Excited leptons at the CERN large hadron collider," Physical Review D, vol. 65, no. 7, 2002.

[25] S. C. İnan, "Exclusive excited leptons search in two lepton final states at the CERN LHC," Physical Review D, vol. 81, no. 11, Article ID 115002, 7 pages, 2010.

[26] O. Çakır, C. Leroy, R. Mehdiyev, and A. Belyaev, "Production and decay of excited electrons at the LHC," The European Physical Journal C, vol. 32, supplement 2, pp. s1-s17, 2004.

[27] A. Belyaev, C. Leroy, and R. Mehdiyev, "Production of excited neutrinos at the LHC," The European Physical Journal C, vol. 41, supplement 2, pp. 1-10, 2005.

[28] E. Boos, A. Vologdin, D. Toback, and J. Gaspard, "Prospects of searching for excited leptons during run II of the Fermilab Tevatron," Physical Review D, vol. 66, no. 1, Article ID 013011, 5 pages, 2002.

[29] D. Acosta, J. Adelman, T. Affolder et al., "Search for excited and exotic electrons in the e $\gamma$ decay channel in $p \bar{p}$ collisions at $\sqrt{s}=1.96 \mathrm{TeV}$," Physical Review Letters, vol. 94, Article ID 101802, 2005.

[30] W. M. Abazo, K. Bloom, and G. R. Snow, "Search for excited electrons in $p \bar{p}$ collisions at $\sqrt{s}=1.96 \mathrm{TeV}$," Physical Review D, vol. 77, no. 9, Article ID 091102, 2008.

[31] P. Banerjee and U. A. Yajnik, "Production and decay rates of excited leptons in a left-right symmetric scenario," Physical Review D, vol. 90, no. 9, Article ID 095023, 2014.

[32] O. Çakır and A. Ozansoy, "Search for excited spin-3/2 and spin1/2 leptons at linear colliders," Physical Review D, vol. 77, no. 3 , Article ID 035002, 2008.

[33] O. Çakır and A. Ozansoy, "Single production of excited spin-3/2 neutrinos at linear colliders," Physical Review D, vol. 79, no. 5, Article ID 055001, 11 pages, 2009.
[34] A. Ozansoy, V. Arı, and V. Çetinkaya, "Search for excited spin3/2 neutrinos at LHeC", Advances in High Energy Physics, vol. 2016, Article ID 1739027, 10 pages, 2016.

[35] S. R. Choudhury, R. G. Ellis, and G. C. Joshi, "Limits on excited spin-3/2 leptons," Physical Review D, vol. 31, no. 9, pp. 23902392, 1985.

[36] D. Spehler, O. J. Éboli, G. C. Marques, S. F. Novaes, and A. A. Natale, "Looking for spin-3/2 leptons in hadronic collisions," Physical Review D, vol. 36, no. 5, pp. 1358-1362, 1987.

[37] F. Almeida, J. Martins Simões, and A. Ramalho, "lepton production at HERA," Nuclear Physics B, vol. 397, no. 3, pp. 502-514, 1993.

[38] F. M. L. Almeida Jr., J. H. Lopes, J. A. Martins Simões, and A. J. Ramalho, "Production and decay of single heavy spin$3 / 2$ leptons in high energy electron-positron collisions," Physical Review D, vol. 53, no. 7, pp. 3555-3558, 1996.

[39] G. Abbiendi, C. Ainsley, P. F. Åkesson et al., "Search for charged excited leptons in $\mathrm{e}^{+} \mathrm{e}^{-}$collisions at $\sqrt{s}=183-209 \mathrm{GeV}$," Physics Letters B, vol. 544, no. 1-2, pp. 57-72, 2002.

[40] G. Aad, B. Abbott, J. Abdallah et al., "Search for new phenomena in events with three or more charged leptons in pp collisions at $\sqrt{s}=8 \mathrm{TeV}$ with the ATLAS detector," Journal of High Energy Physics, vol. 2015, no. 8, article 138, 2015.

[41] H. Gittleson, T. Kirk, M. Murtagh et al., "Search for excited muons," Physical Review D, vol. 10, no. 5, article 1379, 1974.

[42] F. Renard, "Limits on masses and couplings of excited electrons and muons," Physics Letters B, vol. 116, no. 4, pp. 264-268, 1982.

[43] W. T. Ford, A. L. Read, J. G. Smith et al., "Experimental test of higher-order QED and a search for excited muon states," Physical Review Letters, vol. 51, no. 4, article 257, 1983.

[44] F. A. Berends and P. H. Daverveldt, "Effects of an excited muon on $\mu+\mu-\gamma$ final states," Nuclear Physics, Section B, vol. 272, no. 1 , pp. 131-144, 1986.

[45] J. A. Grifols and S. Peris, "Limits on masses of excited electrons and muons from neutrino scattering off electrons," Physics Letters B, vol. 168, no. 3, pp. 264-266, 1986.

[46] B. Adeva, O. Adriani, M. Aguilar-Benitez et al., "Mass limits for excited electrons and muons from $Z^{0}$ decay," Physics Letters $B$, vol. 247, no. 1, pp. 177-184, 1990.

[47] A. Abulencia, D. Acosta, J. Adelman et al., "Search for excited and exotic muons in the $\mu \gamma$ decay channel in $p \bar{p}$ collisions at $\sqrt{s}=1.96$ TeV," Physical Review Letters, vol. 97, no. 19, Article ID 191802, 7 pages, 2006.

[48] V. M. Abazov, B. Abbott, M. Abolins et al., "Search for excited muons in $p \bar{p}$ collisions at $\sqrt{s}=1.96 \mathrm{TeV}$," Physical Review D, vol. 73, no. 11, Article ID 111102, 2006.

[49] G. Aad, T. Abajyan, B. Abbott et al., "Search for excited electrons and muons in $\sqrt{s}=8 \mathrm{TeV}$ proton-proton collisions with the ATLAS detector," New Journal of Physics, vol. 15, Article ID 093011, 2013.

[50] The ATLAS Collaboration, "A search for an excited muon decaying to a muon and two jets in $p p$ collisions at $\sqrt{s}=8 \mathrm{TeV}$ with the ATLAS detector," New J. Phys, vol. 18, no. 7, Article ID 073021, 2016.

[51] V. Khachatryan, A. M. Sirunyan, A. Tumasyan et al., "Search for excited leptons in proton-proton collisions at $\sqrt{s}=8 \mathrm{TeV}$," Journal of High Energy Physics, vol. 2016, no. 3, article 125, 2016.

[52] C. Adolphsen, M. Barone, B. Barish et al., "The International Linear Collider Technical Design Report - Volume 3.II: Accelerator Baseline Design," https://arxiv.org/abs/1306.6328. 
[53] J. P. Delahaye, C. Ankenbrandt, A. Bogacz et al., "Enabling intensity and energy frontier science with a muon accelerator facility in the U.S.," https://arxiv.org/abs/1308.0494.

[54] J. L. Abelleira Fernandez, C. Adolphsen, A. N. Akay et al., "A large hadron electron collider at CERN. Report on the physics and design concepts for machine and detector," Journal of Physics G, vol. 39, Article ID 075001, 2012.

[55] O. Brüning and M. Klein, "The large hadron electron collider," Modern Physics Letters A, vol. 28, no. 16, Article ID 1330011, 2013.

[56] M. Aicheler, P. Burrows, M. Draper et al., "A multi-TeV linear collider based on CLIC technology: CLIC conceptual design report," KEK Report JAI-2012-001, 2012-1, PSI-12-01, SLAC-R985, CERN, Geneva, Switzerland, 2012, https://edms.cern.ch/ ui/file/1234244/7/CERN-2012-007.pdf.

[57] J. P. Delahaye, E. Adli, S. J. Gessner et al., "A beam driven plasma-wakefield linear collider from Higgs factory to multi$\mathrm{TeV}$," in Proceedings of the 5th International Particle Accelerator Conference (IPAC '14), pp. 3791-3793, Dresden, Germany, June 2014.

[58] FCC, https://fcc.web.cern.ch.

[59] M. Bicer, H. Duran Yildiz, I. Yildiz et al., "First look at the physics case of TLEP," Journal of High Energy Physics, vol. 2014, no. 1, article 164, 2014.

[60] F. Zimmerman, "Challenges for highest energy circular colliders," in Proceedings of the KEK Accelerator Seminar, Tsukuba, Japan, 2014.

[61] Y. C. Acar, A. N. Akay, S. Beser et al., "FCC based LeptonHadron and photon-hadron colliders: luminosity and physics," in Proceedings of the 2nd Annual Meeting of the Future Collider Study (FCC Week 2016), Rome, Italy, April 2016.

[62] Y. C. Acar, U. Kaya, B. B. Oner, and S. Sultansoy, "FCC based ep and $\mu$ p colliders," https://arxiv.org/abs/1510.08284.

[63] Y. C. Acar, A. N. Akay, S. Beser et al., "FCC based LeptonHadron and Photon-Hadron colliders: luminosity and physics," https://arxiv.org/abs/1608.02190.

[64] U. Baur, M. Spira, and P. M. Zerwas, "Excited-quark and -lepton production at hadron colliders," Physical Review D, vol. 42, no. 3, pp. 815-824, 1990.

[65] A. Belyaev, N. D. Christensen, and A. Pukhov, "CalcHEP 3.4 for collider physics within and beyond the Standard Model," Computer Physics Communications, vol. 184, no. 7, pp. 17291769, 2013.

[66] A. Pukhov, "CalcHEP 2.3: MSSM, structure functions, event generation, batchs, and generation of matrix elements for other packages," https://arxiv.org/abs/hep-ph/0412191.

[67] A. Pukhov, E. Boos, M. Dubinin et al., "CompHEP—a package for evaluation of Feynman diagrams and integration over multi-particle phase space. User's manual for version 33," https://arxiv.org/abs/hep-ph/9908288. 

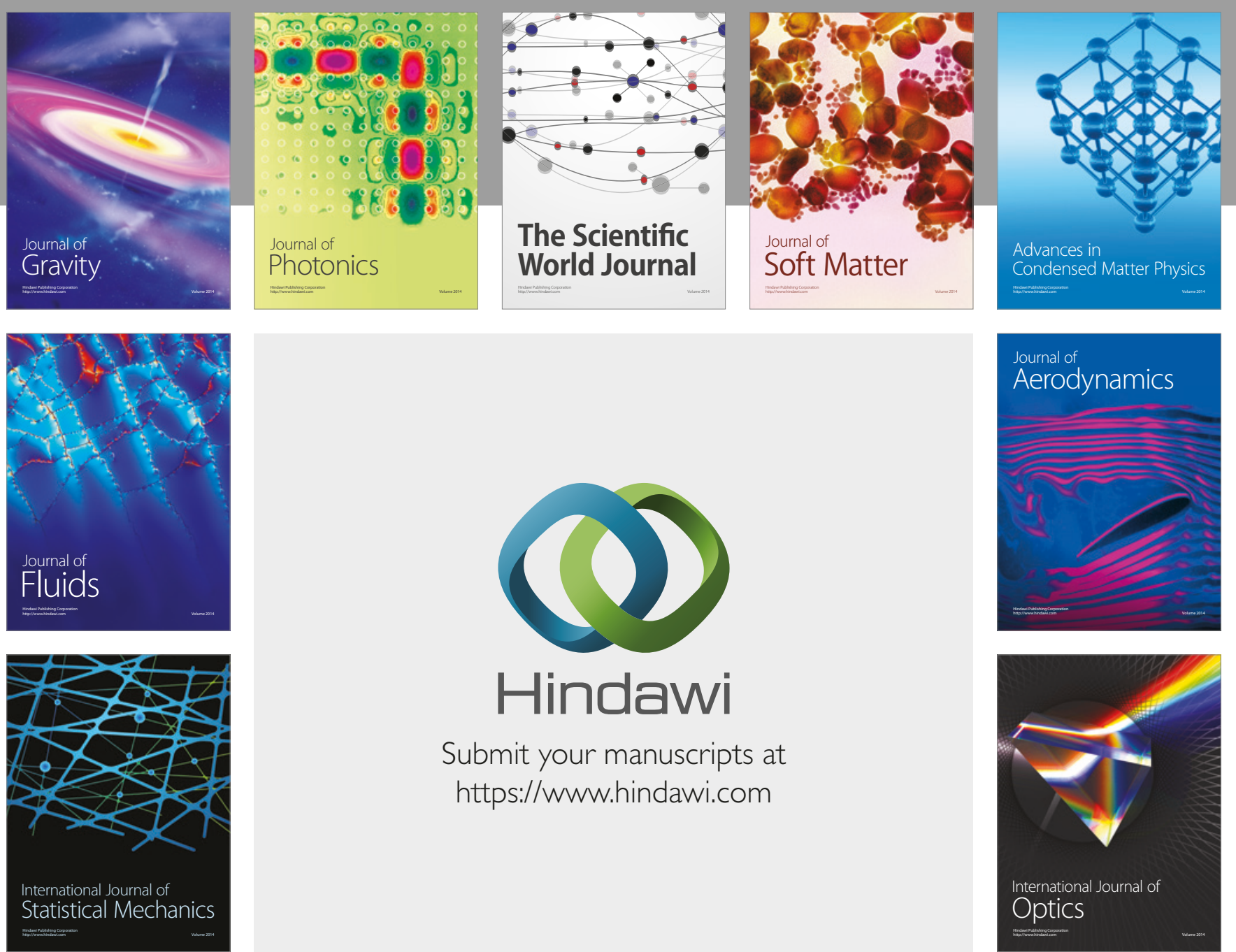

Submit your manuscripts at

https://www.hindawi.com
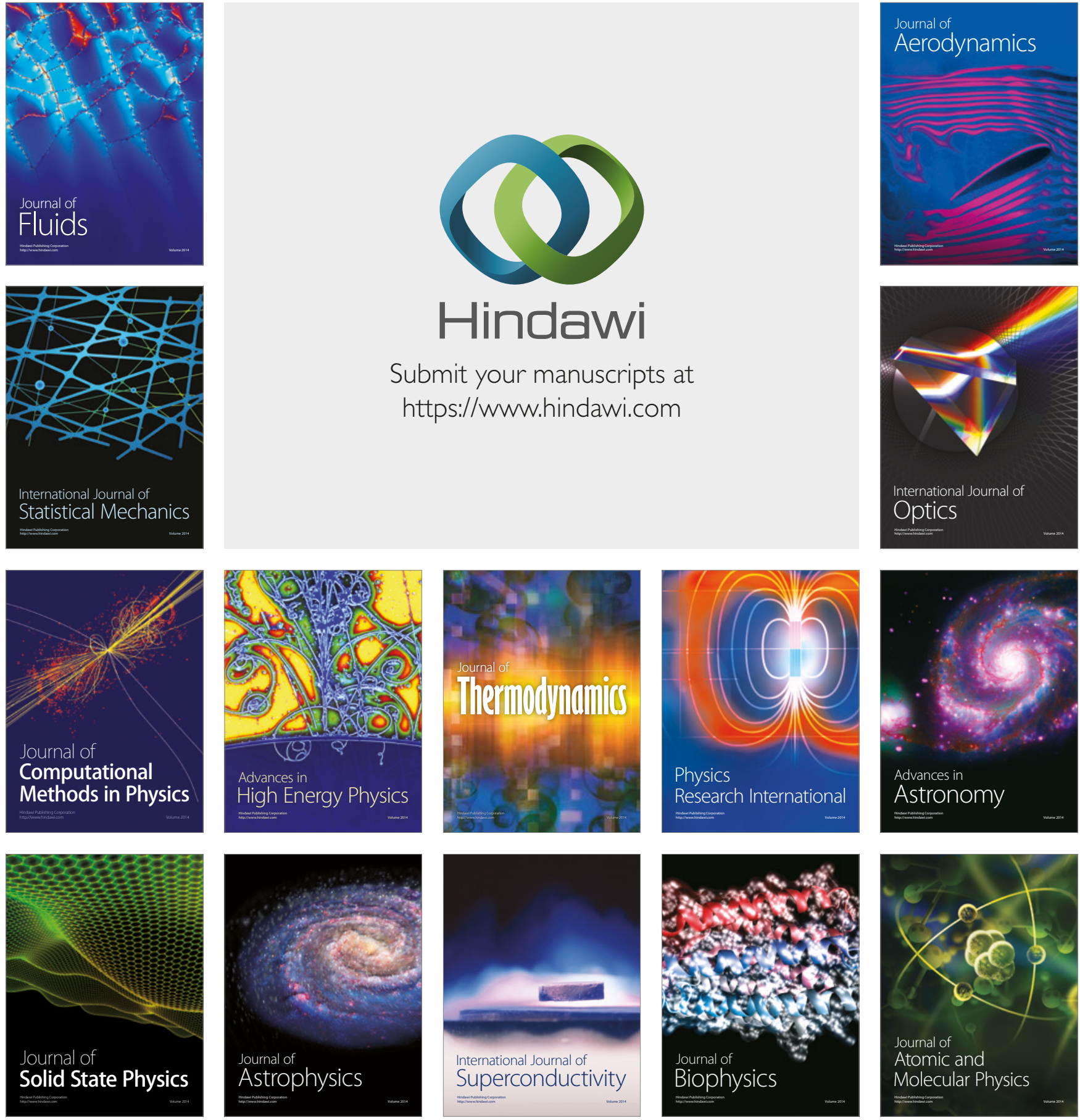